\title{
Pharmacokinetics of KA2237, a novel selective inhibitor of PI3K- $\beta$ and PI3K- $\delta$, in patients: A first-in-human study using PK modelling to predict drug concentrations during dose escalation
}

\author{
James Dow ${ }^{1}$, Graham Trevitt ${ }^{2}$, Elisabeth Bone ${ }^{1}$, Kemal Haque $^{1}$, and Loretta Nastoupil ${ }^{3}$ \\ ${ }^{1}$ Karus Therapeutics Ltd \\ ${ }^{2}$ XenoGesis Ltd \\ ${ }^{3}$ The University of Texas MD Anderson Cancer Center
}

June 25, 2021

\begin{abstract}
Aims: KA2237, an oral, potent and selective, inhibitor of the PI3K $\beta$ and $\delta$ isoforms, was evaluated for safety, tolerability and pharmacokinetics (PK) in patients with B-cell lymphoma. KA2237 is metabolised by CYP3A4/5 but also demonstrated mechanism-based inhibition (MBI) of CYP3A4/5. An MBI mechanistic dynamic model was used to predict drug accumulation after repeat dosing of KA2237. This model, along with clinical safety data, was used to guide safe dose escalation. Methods: An open-label, single arm, dose escalation study was carried out in patients, dosed orally with KA2237 at 50, 100,200 and 400 mg once daily. Complete plasma profiles were obtained on Day 1 and Day 14 of dosing and pre-dose (Cmin) samples were obtained on Days 2-7. The MBI model was validated and used to calculate drug levels and predict potential drug accumulation during dose escalation. Results: KA2237 elimination half-life was around 20-30 h, compatible with once daily dosing regimens. The accumulation of KA2237 was around 4-fold after the highest dose of $400 \mathrm{mg}$ and around 3-fold after administration of $200 \mathrm{mg}$, which is considered the maximum tolerated dose (MTD). The MBI model accurately predicted this accumulation. Conclusions: Drugs that demonstrate MBI and potential auto-inhibition can be successfully developed, provided that models are developed to assess the extent of accumulation prior to the start of FIH clinical studies. This, along with the close monitoring of drug levels and clinical safety data can be used to guide dose escalation and lead to the safe conduct of clinical studies.
\end{abstract}

\section{Hosted file}

KA2237 clin PK modelling publication JD 10June2021_final_Text.docx available at https:// authorea.com/users/421865/articles/527716-pharmacokinetics-of-ka2237-a-novel-selectiveinhibitor-of-pi3k-\%CE\%B2-and-pi3k-\%CE\%B4-in-patients-a-first-in-human-study-using-pkmodelling-to-predict-drug-concentrations-during-dose-escalation

\section{Hosted file}

KA2237 clin PK modelling publication figures_final.docx available at https://authorea.com/ users/421865/articles/527716-pharmacokinetics-of-ka2237-a-novel-selective-inhibitor-ofpi3k-\%CE\%B2-and-pi3k-\%CE\%B4-in-patients-a-first-in-human-study-using-pk-modelling-topredict-drug-concentrations-during-dose-escalation 